\title{
Collagen deposition in liver disease
}

\author{
J. O'D. MCGEE
}

From the Department of Pathology, Radcliffe Infirmary, Oxford

Collagen forms a small component of the total protein of normal liver, but in diseases which lead to cirrhosis and in cirrhosis itself it is deposited in large amounts throughout the organ. This leads to structural disruption of normal liver architecture (Fig. 1). The effects of this are the creation of portosystemic shunts within the liver (Hase, 1968) and perhaps the formation of a connective tissue diffusion barrier in the space of Disse between hepatocytes and their vascular supply.

This paper presents evidence that collagen deposition in liver disease is due, at least in part, to increased collagen synthesis. Those liver cells which are responsible for collagen synthesis and the regulation of the synthesis of this protein in the diseased liver are also briefly discussed.

\section{Collagen synthesis}

Collagen synthesis may readily be studied in mouse liver after one dose of carbon tetrachloride. After one dose the hepatocytes in the centre of the liver lobule undergo necrosis, the necrotic cells are then removed, and by the fifth day the centre of the lobule has become repopulated with new hepatocytes derived from the periportal area. During the healing of this lesion an increased amount of collagen can usually be visualized in the centre of the lobule three to five days after poisoning. These fibres disappear very rapidly and the liver is morphologically normal on the sixth to seventh day of the experiment.

In this model the rate of collagen synthesis is increased 24 hours after injury, reaches a maximum

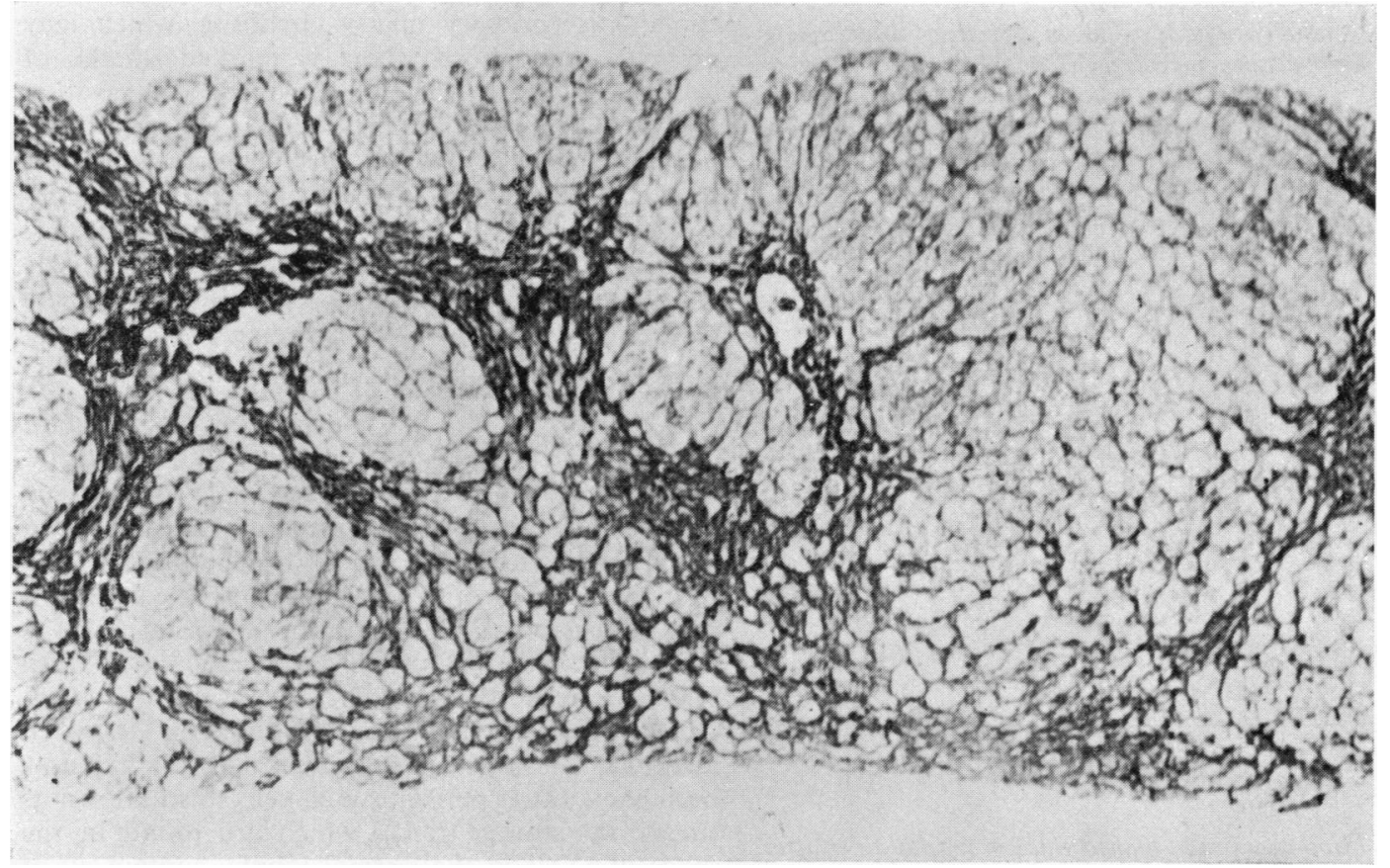

FIG. 1 Percutaneous needle biopsy of liver showing alcoholic cirrhosis with pronounced connective tissue deposition throughout the liver parenchyma. Reticulin stain. 
on the third to fourth day, and returns to normal by the sixth day (Fig. 2). The level of prolyl hydroxylase shows a similar response after injury (Fig. 3). This enzyme, which synthesizes the hydroxyproline of collagen, is considered to be almost unique to collagen synthesizing cells. These data indicate, therefore, that those fibres deposited in the centre of the lobule may have their genesis in increased collagen synthesis. In human liver disease direct evidence of increased collagen synthesis is difficult to obtain because the usual isotopic techniques for measuring collagen synthesis cannot effectively be

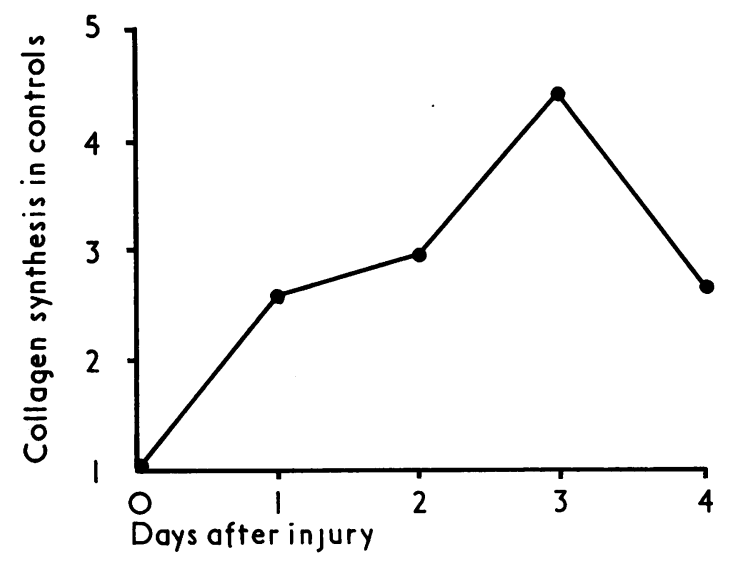

FIG. 2 Collagen synthesis in mouse liver after single dose of carbon tetrachloride $\left(\mathrm{CCl}_{4}\right)$. Male mice weighing about $20 \mathrm{~g}$ were given by oesphageal tube $0.2 \mathrm{ml}$ of a $40 \%$ solution of $\mathrm{CCl}_{4}$ in liquid paraffin. Collagen synthesis in liver was measured as described by McGee et al. (1973). Each point is average of 2 determinations in 2 separate livers. Rate of collagen synthesis in poisoned animals expressed as a ratio of that found in control normal livers

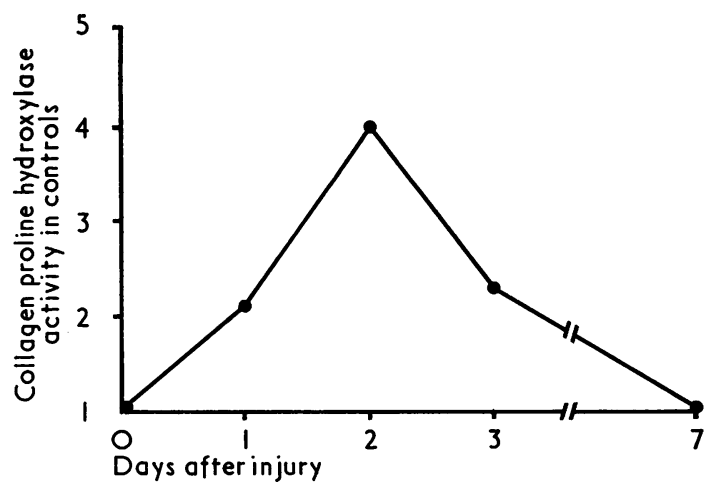

FIG. 3 Collagen prolyl hydroxylase in normal liver after carbon tetrachloride injury. Mice were poisoned as outlined in legend to Fig. 2. Prolyl hydroxylase was measured as detailed by McGee et al. (1973). Results expressed as in Fig. 2
Table I Prolyl hydroxylase in normal liver and in cirrhosis. Enzyme estimations performed as described by $\mathrm{McGee}$ et al. (1974)

\begin{tabular}{lcc}
\hline Diagnosis & & Prolylhydroxylase (cpm/mg protein) \\
\cline { 1 - 1 } Normal & & $1158(726,893,1459,1553)$ \\
Gilbert's disease* & & $1310(946,1674)$ \\
Cirrhosis & & $3585-9588(3585,6000,9144,9366$ \\
& $9588)$
\end{tabular}

*Gilbert's disease is characterized by a defect in the liver's ability to handle bilirubin. It is not associated with collagen deposition.

applied to the small amounts of tissue (about 10-12 mg wet weight) obtained by percutaneous needling of the liver. However, it can be shown in human cirrhosis (Table I) and in diseases which lead to cirrhosis (unpublished data) that the hepatic level of prolyl hydroxylase is raised, suggesting that the rate of collagen synthesis is also increased (McGee et al., 1974).

The site at which connective tissue synthesis occurs in liver has been investigated by autoradiography using ${ }^{35} \mathrm{~S}$-sulphate, which is a precursor of the sulphated glycosaminoglycans of ground substance. In a form of non-progressive hepatic fibrosis (congenital hepatic fibrosis) only occasional sinusoidal cells and a few cells in fibrous bands were active in the uptake of ${ }^{35} \mathrm{SO}_{4}$. In other diseases such as alcoholic hepatitis and non-suppurative destructive cholangitis (primary biliary cirrhosis), which may progress to cirrhosis, there is marked uptake of isotope at the sites of connective tissue deposition and in portal tracts (Figs. 4, 5). Cells related to hepatic sinusoids rather surprisingly also show marked uptake of isotope (Fig. 6). It can be concluded from these results that sulphated glycosaminoglycan synthesis is increased in aggressive liver disease and that this occurs not only where connective tissue is obviously being laid down but also in hepatic sinusoids.

\section{Liver cells responsible for collagen formation}

The nature of the cell type responsible for collagen formation in the liver has been investigated by a variety of methods (Popper and Udenfriend, 1970). Electron microscopic investigations have shown that connective tissue deposition in portal tracts is associated with typical fibroblastic activity. Until relatively recently, however, cells with the ultrastructural characteristics of fibroblasts had not been identified within the liver lobule itself. It has now been shown that perisinusoidal cells (also known as Ito or fat storage cells), which are found in the space of Disse in the normal lobule, contain all of the subcellular machinery needed for the synthesis of proteins for export-for example, a well developed rough endoplasmic reticulin and Golgi apparatus 


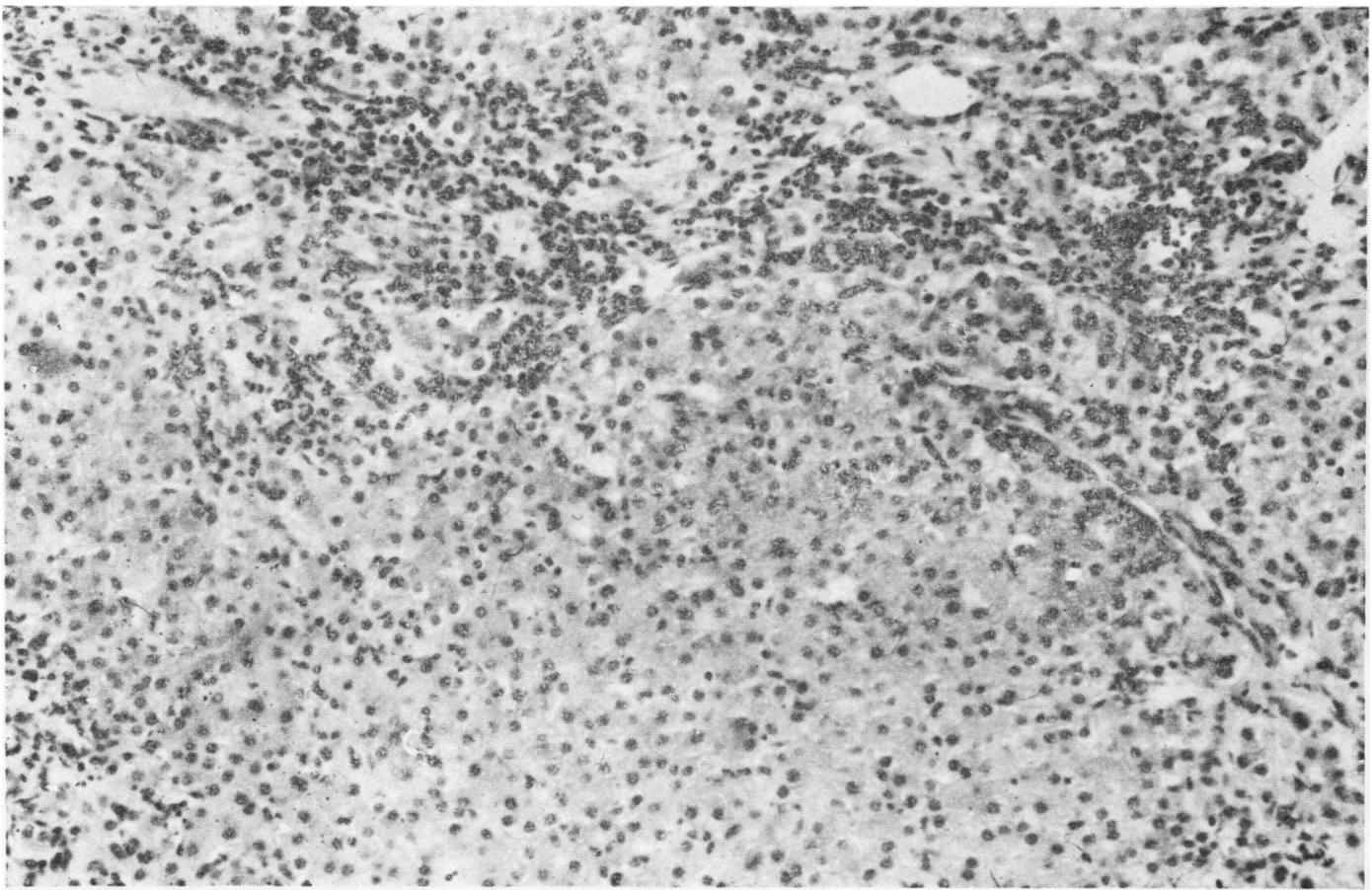

FIG. 4 Histological appearance of liver in non-suppurative destructive cholangitis (primary biliary cirrhosis). $\frac{0}{2} \overrightarrow{0}$ Inflammatory cell infiltration and increased connective tissue formation in a portal tract (upper half of figure)

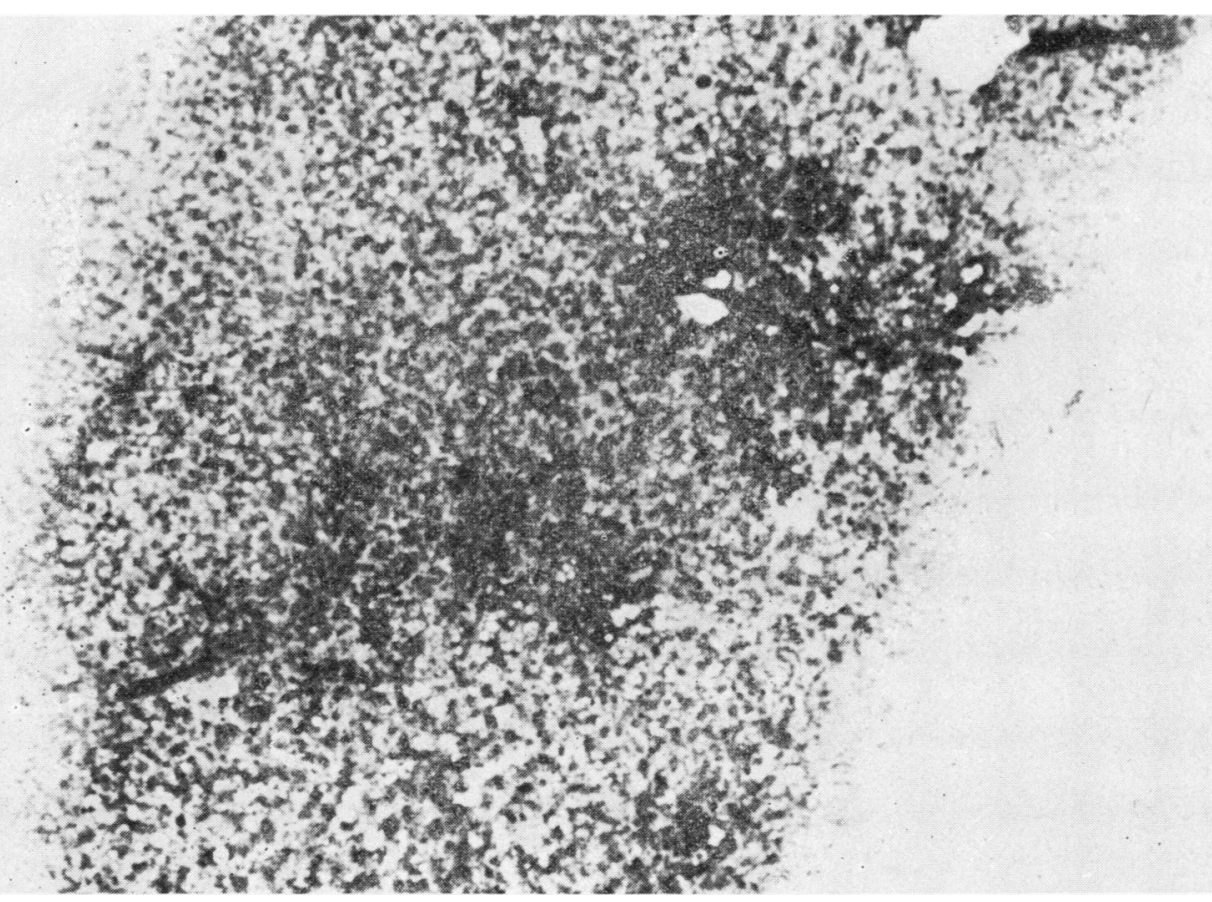

FIG. 5 Autoradiograph of ${ }^{35} \mathrm{SO}_{4}$ uptake in same liver as in Fig. 4. Shows marked uptake of isotope in portal tracts where connective tissue is being laid down (McGee et al., 1974) 


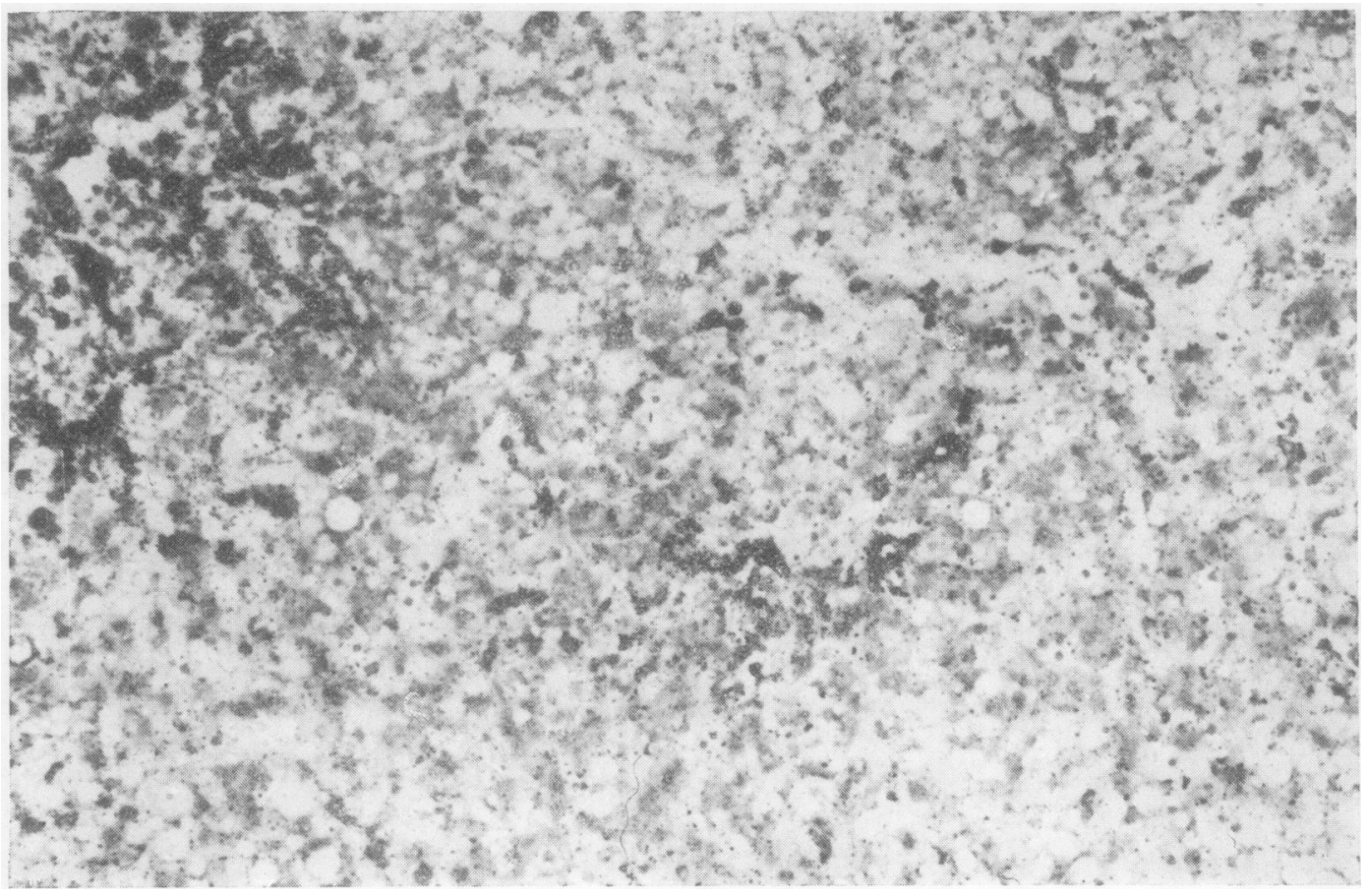

FIG. 6 Autoradiograph of ${ }^{35} \mathrm{SO}_{4}$ uptake in same liver as in Fig. 5. Higher power magnification shows that isotope्ड. is being utilized by cells related to hepatic sinusoids

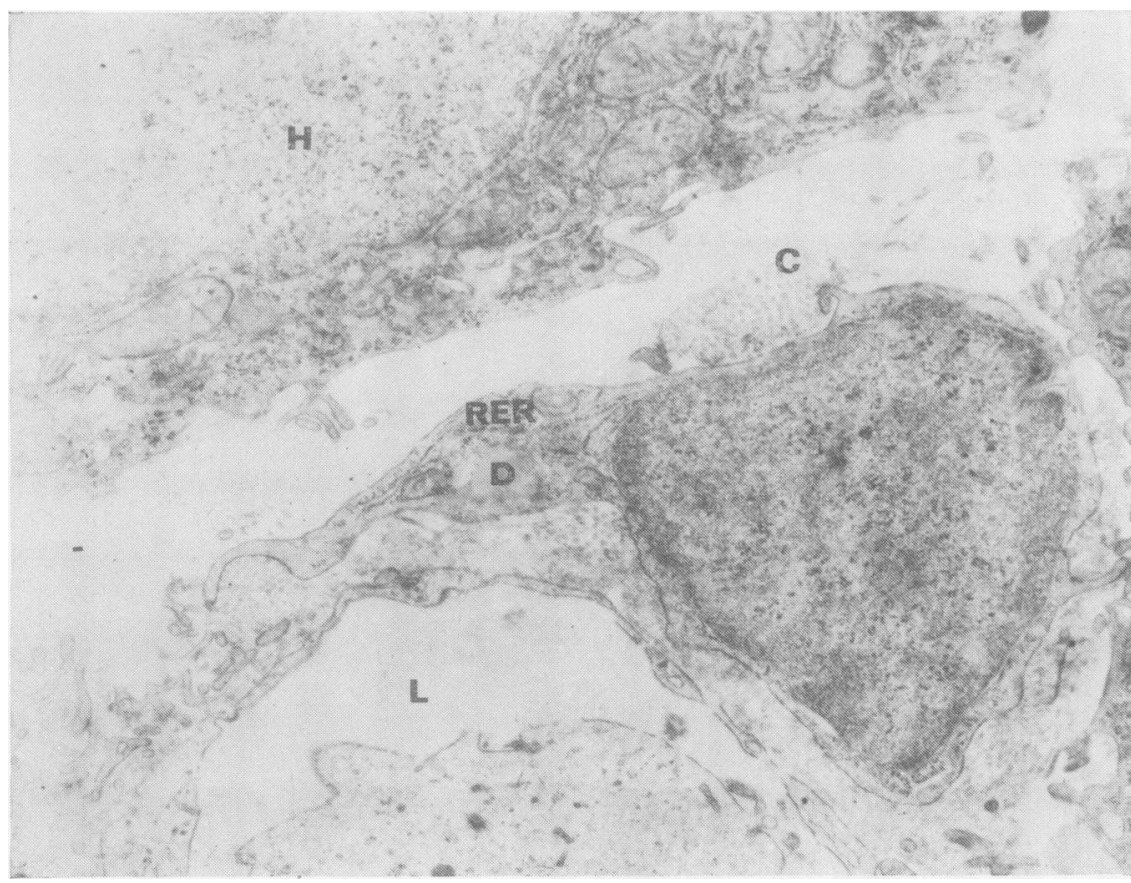

FIG. 7 Electron micrograph of normal human hepatic sinusoid. Perisinusoidal cell with well developed rough endoplasmic reticulum (RER) lies in space of Disse and is closely associated with collagen fibrils $(C)$. It is separated from sinusoidal lumen $(L)$ by sinusoidal cells. $H=$ hepatocyte. $D=$ lipid droplet 


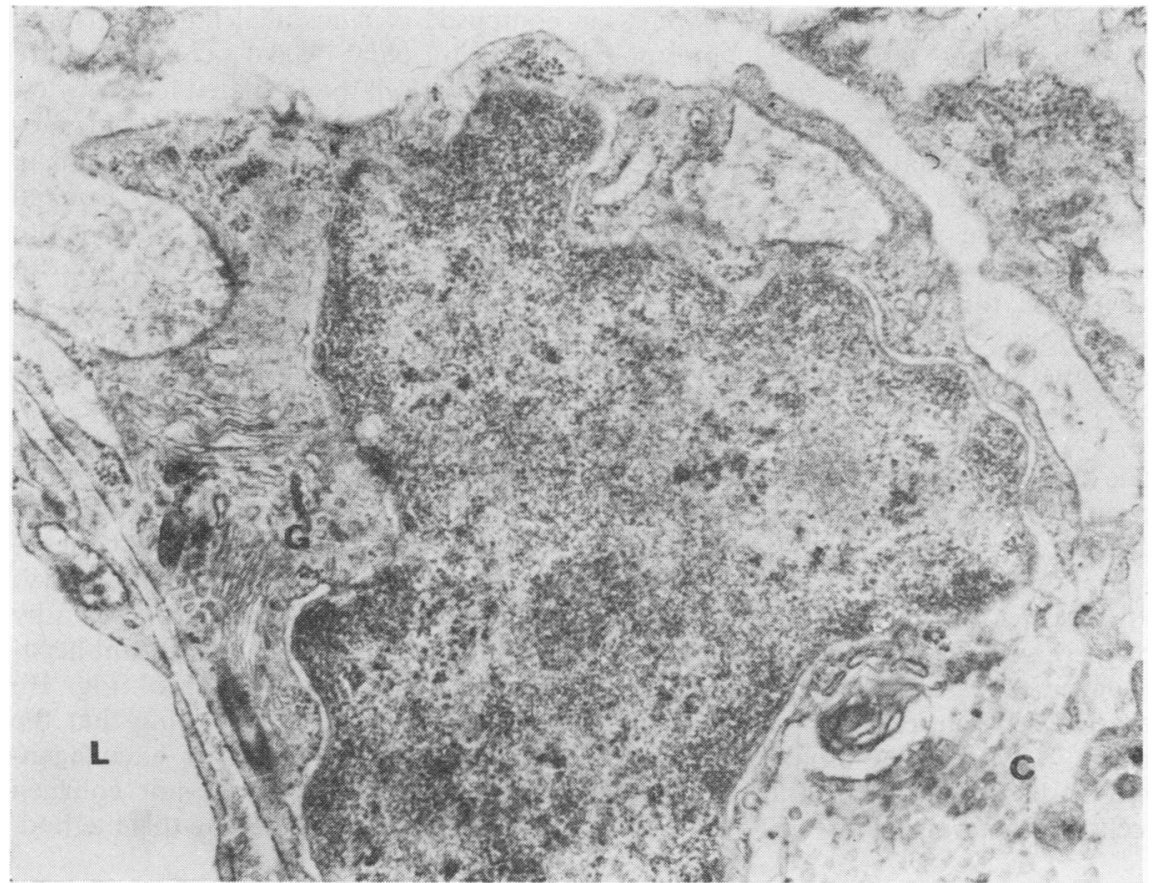

FIG. 8 Electron micrograph of perisinusoidal $\vec{\circ}$ cell in normal human liver. Perisinusoidal cell is $\vec{\omega}$ closely associated with collagen fibrils $(C)$ and 을 has prominent Golgi $\omega$ apparatus (G)

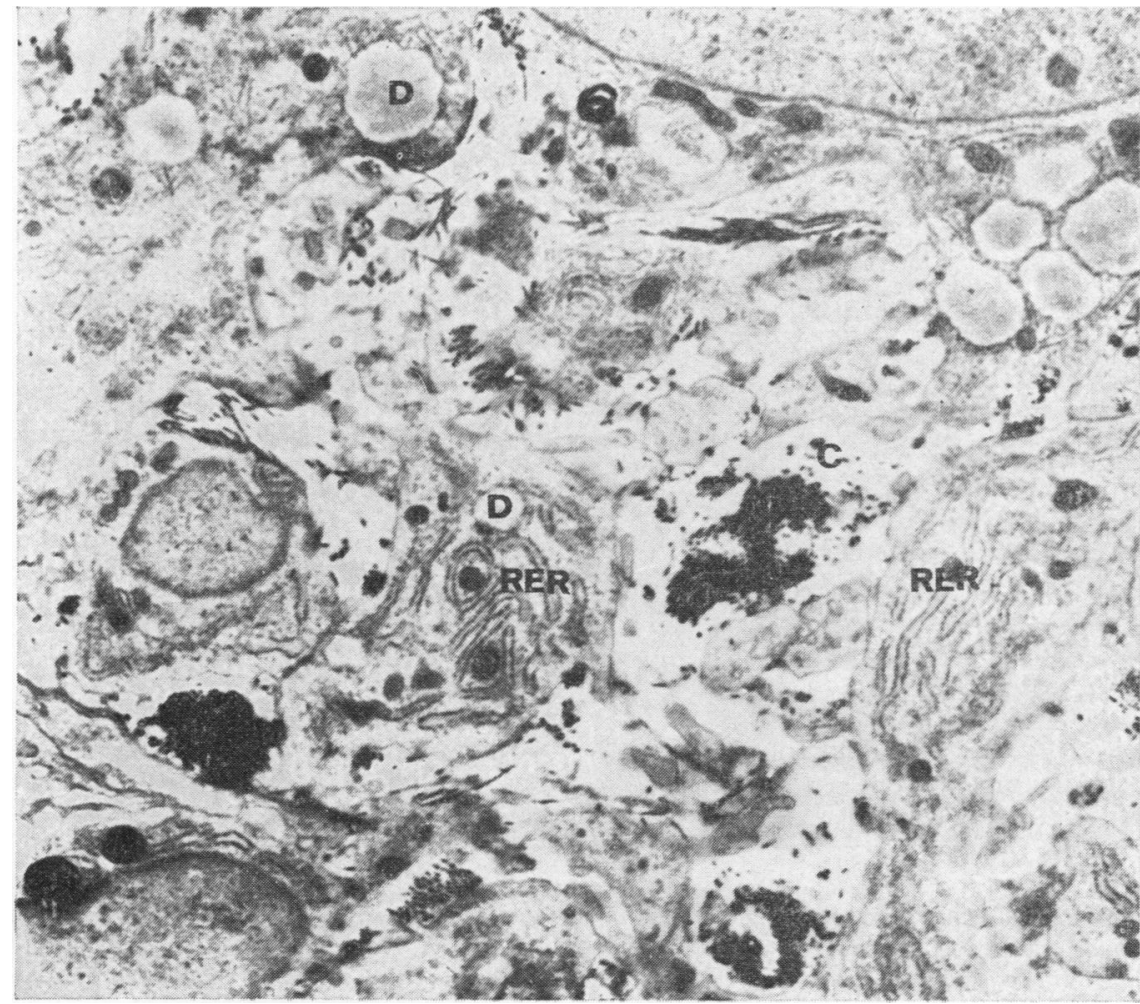

FIG. 9 Electron micrograph of healing zone in mouse liver 4 days after single dose of $40 \% \mathrm{CCl}_{4}$ 항 in liquid paraffin. Numer- 3 ous cells throughout the area contain a well developed rough endoplasmic reticulum (RER) and are associated with numerous collagen fibrils $(C)$. Many cells contain lipid droplets (D) 
(Figs. 7 and 8). In the normal liver these cells may be intimately associated with collagen fibrils (Fig. 8). In addition these cells increase in number and size in zones where collagen is deposited in experimental liver injury (Fig. 9). This, then, is prima facie evidence that perisinusoidal cells may be the intralobular liver fibroblasts. The presence of prominent lipid droplets within these cells (Fig. 7) has been construed as evidence for a fat storage function. It should be noted, however, that collagenproducing cells in many situations contain intracytoplasmic lipid droplets (McGee and Patrick, 1972).

Claims have been made that hepatocytes have a collagen productive function (Rojkind and MartinezPalomo, 1976). This is based mainly on the finding that isolated hepatocytes contain prolyl hydroxylase. The isolated cells used in these experiments, however, were only $95 \%$ pure and perhaps a contaminating cell was the source of the enzyme measured, because isolated hepatic mesenchymal cells entirely free of hepatocytes also contain prolyl hydroxylase (Shaba et al., 1973). More recently it has been shown that isolated mesenchymal cells (a heterogeneous cell population composed of sinusoidal lining cells and presumably perisinusoidal cells) contain more prolyl hydroxylase per cell than isolated hepatocytes. Furthermore, when mesenchymal cells and hepatocytes are isolated from regenerating rat liver in which collagen synthesis is increased isolated mesenchymal cells contain at least eight times more prolyl hydroxylase than isolated hepatocytes (Martin and Patrick, unpublished). Since it is difficult to isolate hepatocytes and mesenchymal cells in an entirely pure form the interpretation of experiments such as these is at present not clear.

Although the evidence available is against a significant collagen biosynthetic role for hepatocytes it has been shown recently that malignant hepatocytes may assume this function. In human hepatomas and in the same tumour induced by an azo dye carcinogen (unpublished) it has been shown by immunohistochemical methods that malignant hepatocytes contain both prolyl hydroxylase (Fig. 10) and collagen antigen (Fig. 11), suggesting that the 'transformed' hepatocyte may assume a collagenproductive function. Neither enzyme nor collagen can be detected in normal liver cells by this method.

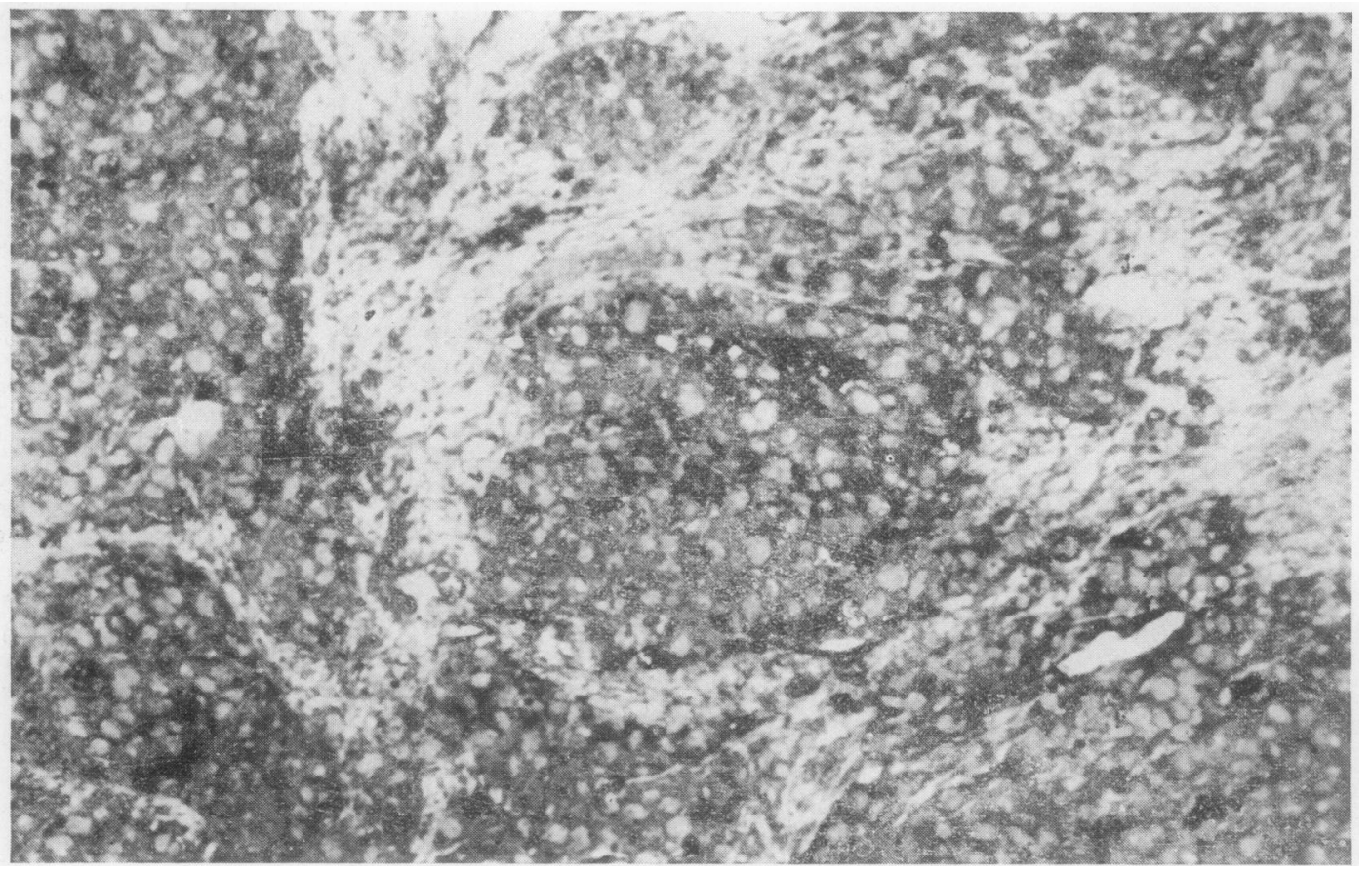

FIG. 10 Prolyl hydroxylase in human hepatoma cells identified by the immunoperoxidase procedure using a specific antibody to prolyl hydroxylase. All tumour cells contain brown reaction product indicating presence of prolyl hydroxylase. The nuclei (appearing as white holes) do not contain enzyme. For details of technique see AlAdnani et al. (1975) 


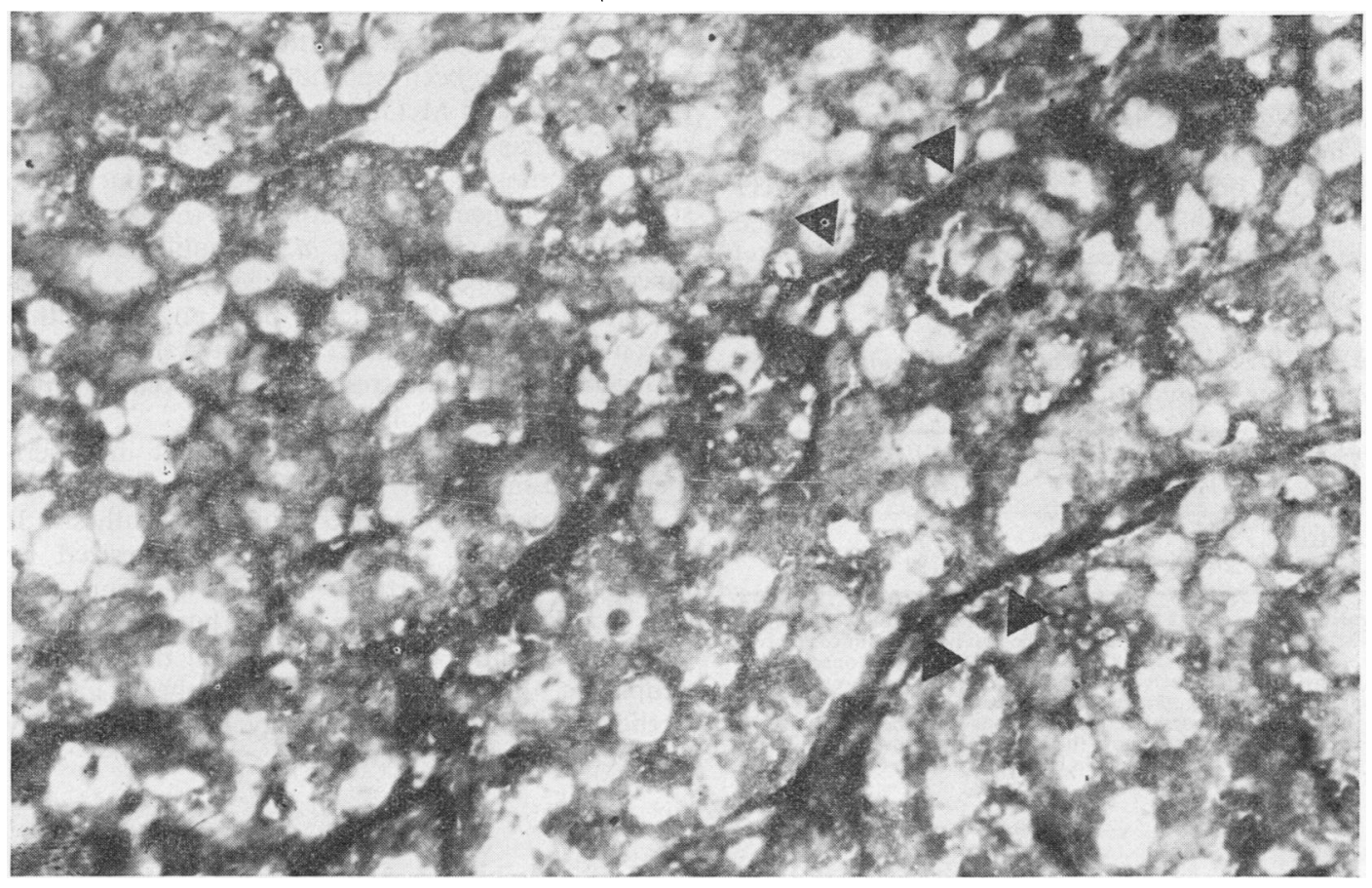

FIG. 11 Collagen antigen in human hepatoma cells identified as in Fig. 10 except that an antibody to neutro $\overrightarrow{0}$ salt soluble collagen was used. Collagen antigen present in the cytoplasm of malignant hepatocytes but not in the nucle Collagen fibres are also stained (arrows)

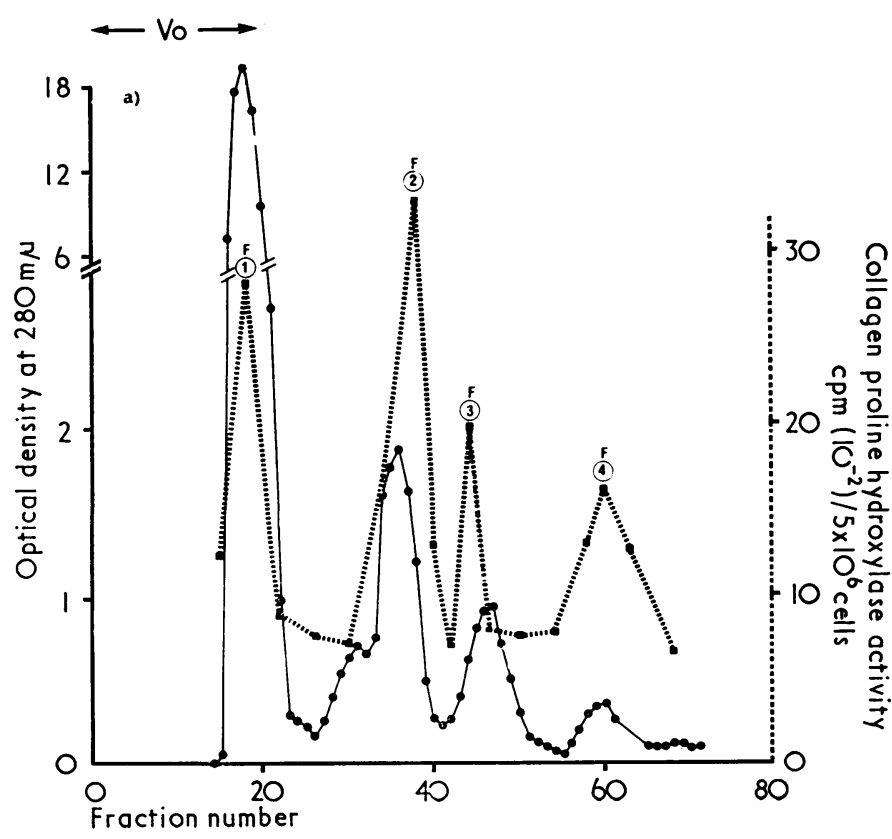

FIG. 12 Isolation of collagen stimulating factors by Sephadex G25 chromatography from mouse liver 3 days after single dose of carbon tetrachloride. Factors labelled $F_{1}-F_{4}(\square$ (IIIII) were isolated from a $15000 \times g$ supernatant derived from livers homogenized in a phosphate buffer (McGee et al., 1973). 욱 The assay for collagen-stimulating $\rightarrow$ factors based on their ability to stimu- 을. late prolyl hydroxylase activity in $\mathrm{N}$ cultured fibroblasts is described by McGee $\Omega$ et al. (1973) with one modification- N namely, the fibroblasts used in this assay N were grown in medium containing $10 \% \mathrm{\omega}$ fetal calf serum and other supplements. fetal calf serum 


\section{Regulation of collagen synthesis}

The regulation of collagen synthesis in collagenproductive diseases such as cirrhosis has only begun to be investigated (McGee et al., 1973). A series of factors can be isolated from experimental liver disease in which collagen synthesis is increased (Table II) which will cause an increase in prolyl

Table II Prolyl hydroxylase and collagen synthesis in mouse liver 3 days after carbon tetrachloride

\begin{tabular}{|c|c|c|}
\hline Treatment & $\begin{array}{l}\text { Prolyl hydroxylase } \\
\left(\text { cpm } \times 10^{-2} / g\right)\end{array}$ & $\begin{array}{l}\text { Collagen synthesis } \\
(\mathrm{cpm} / \mathrm{g})\end{array}$ \\
\hline $\begin{array}{l}\mathrm{Nil}_{\text {(control) }} \\
\mathrm{CCl}_{4}\end{array}$ & $\begin{array}{r}745 \\
2448\end{array}$ & $\begin{array}{r}510 \\
1731\end{array}$ \\
\hline
\end{tabular}

*Two mice were given $0.2 \mathrm{ml}$ of $40 \% \mathrm{CCl}_{4}$ in olive oil by eosphageal tube. Three days later two normal and the two poisoned mice received $5 \mu \mathrm{Ci}$ of ${ }^{14} \mathrm{C}$-proline (SA $285 \mathrm{mCi} / \mathrm{mMol}$ ) intraperitoneally two hours before killing by cerivcal dislocation. All animals were starved overnight before killing. Prolyl hydroxylase and collagen synthesis were measured in the same livers by methods described elsewhere by McGee et al. (1973). Each value is the mean enzyme level and collagen synthetic rate of the two animals in each group. hydroxylase in fibroblast cultures (Fig. 12). Similar factors have also been isolated from human cirrhotic tissue (Smith and McGee, unpublished data). More recently it has been shown that the effluents from these columns contain four factors which also stimulate collagen chain synthesis in the same tissue culture system (Fallon et al., unpublished data). Since these collagen stimulating factors are not present in normal liver it has been postulated that they may play a role in the regulation of the increased collagen biosynthetic response observed in liver injury (McGee et al., 1973).

In conclusion, there is evidence that in experimental and human liver disease collagen deposition is due in part to increased collagen synthesis. It seems likely that this protein is synthesized by fibroblasts in portal tracts and by perisinusoidal cells in non-neoplastic liver disease but that hepatocytes may assume this function when they become neoplastic. Finally, collagen biosynthesis in precirrhotic and cirrhotic liver may be controlled by collagen stimulating factors. 